expressed reluctance to compress deeply for fear of harming the victims.

Conclusion Training compressions were better quality. The results show the quality of chest compressions delivered by lay bystanders in actual cases, and highlights depth as an area of concern that could improve with training enhancement.

Conflict of interest None

Funding Ministry of Health grant

\section{EXPLAINING VARIATION IN RATES OF NON-TRANSPORT BETWEEN EMERGENCY AMBULANCE SERVICES}

A O'Cathain*, R Jacques, T Stone, EL Knowles, L Bishop-Edwards. School of Health and Related Research (SHARR), University of Sheffield, UK

\subsection{6/10.1136/bmjopen-2018-EMS.12}

Aim In England in 2017ambulance services responded to around 11 million calls. Half of patients were not transported to hospital. $11 \%$ received telephone advice only and $38 \%$ were discharged at scene. For the ten large regional ambulance services, rates of calls ending in telephone advice varied between $5 \%$ and $17 \%$. Rates of discharge at scene varied between $23 \%$ and $51 \%$. The aim was to explain variation in non-conveyance rates between ambulance services

Method A sequential mixed methods study: a qualitative interview study of managers and paramedics (totalling 49 interviews) followed by analysis of one month of routine data from each ambulance service (6 15618 calls).

Results Interviewees identified factors they perceived affected non-transport. Rates of discharge at scene were associated with patient-level factors e.g. age, deprivation and skill level of attending crew. However, variation between ambulance services remained after adjustment for patient-level factors. Variation was explained by ambulance service-level factors: $\%$ of calls attended by paramedics with extended skills (odds ratio 1.05 (95\% CI: 1.04 to 1.07)), the perception of ambulance service staff that paramedics with extended skills were established and valued within the workforce (odds ratio 1.84 (1.45, 2.33), and the perception of ambulance service staff that senior management was risk averse regarding non-conveyance within an ambulance service (odds ratio $0.78(0.63,0.98)$ ).

Conclusion Ambulance service management can take actions to reduce a variation in practice.

Conflict of interest None

Funding National Institute of Health Research.

\section{A NOVEL APPROACH TO HIGH PERFORMANCE CARDIOPULMONARY RESUSCITATION EDUCATION IN A DANISH AMBULANCE SERVICE}

M Betzer*. Falck Emergency - Zealand Region of Denmark

\subsection{6/10.1136/bmjopen-2018-EMS.13}

Aim Out of hospital cardiac arrest (OHCA) is a medical emergency where immediate treatment with cardiopulmonary resuscitation (CPR) are known to be paramount for survival. For professionals, international guidelines recommend high-performance CPR (HP-CPR) along with advanced life support. Currently, HP-CPR is not formally taught nor implemented in Denmark. This study evaluates a novel approach to HP-CPR education in a Danish ambulance service.
Method A two-day intensive course consisting of theoretical presentations and practical supervised workshops was planned and completed. Pre- and post-course, all participants completed a CPR cycle on a mannequin measuring data on CPR performance followed by a multiple-choice test and a self-efficacy questionnaire. The primary outcome was before and after evaluation of individual non-technical skills within resuscitation, the secondary outcome was before and after evaluation of technical skills through CPR performance.

Results In total, 23 EMS providers completed the course. Individual self-efficacy in resuscitation capabilities increased from a mean (SD) $8,09(0,80)$ to $9,3(0,59)(\mathrm{p}<0,001)$ on a $0-10$ score with 0 being inadequate. Resuscitation knowledge improved from a mean (SD) of $76,77 \%(14,4)$ to $90,89 \%$ $(4,9) \quad(\mathrm{p}<0,002)$. CPR performance improved from a mean (SD) $85,52 \%(23,7)$ to $92,70 \%(13)(p=0,126)$.

Conclusion This study suggests that our novel approach to HP-CPR education is advantageous as we found a significant increase in self-efficacy and CPR knowledge along with a nonsignificant increase in CPR performance. Future studies should investigate whether Danish HP-CPR trained EMS providers improve 30 day survival in real-life OHCA.

Conflict of interest None

Funding None

\section{THE USE OF PREHOSPITAL 12-LEAD ELECTROCARDIOGRAMS IN ACUTE STROKE PATIENTS}

1,2S Munro*, 'Debbie Cooke, 'Mark Joy, ${ }^{3}$ Tom Quinn. ${ }^{1}$ University of Surrey, Guildford, UK; ${ }^{2}$ South East Coast Ambulance Service NHS Foundation Trust, Crawley, UK; ${ }^{3}$ Kingston University and St George's, University of London, London, UK

\subsection{6/10.1136/bmjopen-2018-EMS.14}

AIM Emergency medical services (EMS) play a vital role in the recognition, management and transportation of acute stroke patients. UK guidelines recommend clinicians consider performing a prehospital 12-lead electrocardiogram (PHECG) in patients with suspected stroke, but this recommendation is based on expert consensus, rather than robust evidence. The aim of this study was to investigate the association between PHECG and modified Rankin scale (mRS). Secondary outcomes included in-hospital mortality, EMS and in-hospital time intervals and rates of thrombolysis received.

Method A multicentre retrospective cohort study was undertaken. The data collection period spanned from 29/12/2013 $30 / 01 / 2017$. Participants were identified through secondary analysis of hospital data routinely collected as part of the Sentinel Stroke National Audit Programme (SSNAP) and linked to EMS clinical records (PCRs) via EMS incident number.

Results PHECG was performed in 558 (48\%) of study patients. PHECG was associated with an increase in mRS (aOR 1.44, 95\% CI: 1.14 to $1.82, \mathrm{p}=0.002$ ) and in-hospital mortality (aOR 2.07, 95\% CI: 1.42 to $3.00, \mathrm{p}=0.0001$ ). There was no association between PHECG and administration of thrombolysis (aOR 0.92, 95\% CI: 0.65 to 1.30, p=0.63). Patients who had a PHECG recorded spent longer under the care of EMS (median 49 vs 43 min, $p=0.007$ ). No difference in times to receiving brain scan (Median 28 with PHECG vs 29 min no PHECG, $\mathrm{p}=0.14$ ) or thrombolysis (median $46 \mathrm{~min}$ vs $48 \mathrm{~min}, \mathrm{p}=0.82$ ) were observed.

Conclusion This is the first study of its kind to investigate the association between PHECG and functional outcome in stroke patients attended by EMS. Although there are limitations in 